\title{
Estimación preliminar de la retención de carbono en raíces finas y mantillo de un renoval de Nothofagus glauca de la precordillera andina de la Región del Maule, Chile
}

\author{
Preliminary estimation of carbon retention in fine roots and litter in a young \\ Nothofagus glauca forest in Maule Region, Chile \\ Ursula Dolla*, Oscar Vallejos ${ }^{b}$, Natalia Bilbao, Carolina Jara

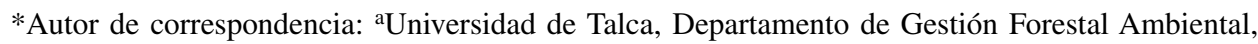 \\ casilla 721-747, Talca, Chile, udoll@utalca.cl \\ bUniversidad de Talca, Departamento de Producción Forestal, Talca, Chile.
}

\begin{abstract}
SUMMARY
In the experimental station "El Picazo" located in the Andes of Maule Region, the quantification of carbon retention by a "RobleHualo" native forest was started. Ten adjacent plots of $1,000 \mathrm{~m}^{2}$ were located in a young forest dominated by Nothofagus glauca, whose basal area and $\mathrm{D}_{\mathrm{g}}$ were $10.8 \mathrm{~m}^{2}$ and $12.3 \mathrm{~cm}$ respectively. Five systematic sampling points were defined in each plot, where litter was sampled with a frame of $0.25 \mathrm{~cm}$, and cylinder-shaped soil samples of $4.5 \mathrm{~cm}$ in diameter were extracted down to 5 , 10 and $20 \mathrm{~cm}$ of the profile. Litter was separated into leaves and branches, and soil samples were washed to separate the roots and classify them into fine roots $(\leq 2 \mathrm{~mm})$ and medium roots $(>2 \mathrm{~mm})$. An accumulation of $12.47 \pm 4.08 \mathrm{Mg}^{-1}$ of litter was determined, where leaves doubled the branch weight. A root-biomass of $20.99 \pm 10.24 \mathrm{Mg} \mathrm{ha}^{-1}$ was found in the upper $20 \mathrm{~cm}$ of the profile. While the fine roots decreased exponentially with depth, the medium ones were distributed uniformly through the soil profile. The carbon retention was estimated to reach $14.72 \mathrm{Mg} \mathrm{ha}^{-1}$, equivalent to $53.97 \mathrm{Mg} \mathrm{ha}^{-1}$ of not emitted $\mathrm{CO}_{2}$.
\end{abstract}

Key words: root-biomass, litter, carbon retention.

\section{RESUMEN}

Se inició la cuantificación del carbono absorbido por un bosque nativo del tipo forestal Roble-Hualo, en el fundo El Picazo ubicado en la precordillera andina de la VII Región del Maule. Dentro de un renoval dominado por Nothofagus glauca, con área basal de $10,8 \mathrm{~m}^{2} \mathrm{ha}^{-1}$ y DMC de $12,3 \mathrm{~cm}$, se delimitaron 10 parcelas contiguas de $1.000 \mathrm{~m}^{2}$. En cada parcela se definieron cinco puntos de muestreo sistemático, donde se recolectó el mantillo en marcos de $0,25 \mathrm{~m}^{2}$, extrayéndose además cilindros de suelo de $4,5 \mathrm{~cm}$ de diámetro, hasta los 5,10 y $20 \mathrm{~cm}$ de profundidad del perfil. El mantillo se separó en hojas y ramas, y las raíces extraídas por lavado de las muestras de suelo se clasificaron según diámetro en finas $(\leq 2 \mathrm{~mm})$ y medianas $(2-5 \mathrm{~mm})$. La acumulación del mantillo alcanzó $12,47 \pm 4,08 \mathrm{Mg} \mathrm{ha}^{-1}$, donde la biomasa de hojas duplicó el peso de las ramas. La biomasa radical en los primeros $20 \mathrm{~cm}$ del perfil totalizó $20,99 \pm 10,24 \mathrm{Mg} \mathrm{ha}^{-1}$, siendo la distribución de raíces finas inversamente proporcional al aumento de la profundidad y la de las raíces medianas uniforme en el perfil. Se estimó que la absorción de carbono ascendió a 14,72 Mg ha-1, equivalente a $53,97 \mathrm{Mg} \mathrm{ha}^{-1}$ de $\mathrm{CO}_{2}$ no emitidos.

Palabras clave: biomasa de raíces, mantillo, absorción de carbono.

\section{INTRODUCCIÓN}

Según IPCC (2001), hacia finales del siglo XXI a nivel mundial habrán aumentado la concentración de $\mathrm{CO}_{2}$ atmosférico, la temperatura media y las precipitaciones. Hay evidencias de que una mayor concentración de $\mathrm{CO}_{2}$ favorece la fotosíntesis de los árboles (Teskey 1997, Tissue et al. 1997, Ellsworth 1999, Myers et al. 1999, Hamilton et al. 2001), de modo que aumentará la biomasa (Groninger et al. 1995, Telewski et al. 1999) y por consiguiente el carbono absorbido por los árboles (Tissue et al. 1997, Ellsworth 1999, Luan et al. 1999).
En Chile existe información restringida de la biomasa en algunos ambientes y tipos forestales, siendo aun más escasas las mediciones de biomasa subterránea y mantillo (Gayoso et al. 2002). Sin embargo, es importante contar con estimaciones precisas de la biomasa del sistema radical (Vogt et al. 1998, Sánchez y Eaton 2001, Claus y George 2005), toda vez que este sistema juega un rol preponderante en la dinámica del carbono bajo el nivel del suelo (Jackson et al. 1997, Rasse et al. 2001, de Koning et al. 2002, Gayoso y Guerra 2005). Tal es el caso para hualo (Nothofagus glauca (Phil.) Krasser) que presenta 351 $\mathrm{Mg} \mathrm{ha}^{-1}$ de biomasa aérea en el sector de Bullileo en la 
provincia de Linares de la VII Región del Maule (Guerra 2001), pero del que no existe información acerca de su producción de hojarasca y raíces.

El hualo es una especie endémica de la zona central de Chile, que crece en las cordilleras de la Costa y de los Andes bajo los $1.200 \mathrm{~m}$ s.n.m., entre los $34^{\circ} 01^{\prime} \mathrm{S}$ y $37^{\circ} 27^{\prime}$ S (Le-Quesne y Sandoval 2001), formando parte del tipo forestal Roble-Hualo (Donoso 1981). Esta formación boscosa ocupa 188 mil hectáreas en la región mediterránea de Chile, de las cuales el 79\% se ubica en la VII Región del Maule (CONAF-CONAMA 1999). Producto de una fuerte presión antrópica, la mayor parte de esta superficie (87\%) (CONAF-CONAMA 1999) está representada por renovales de hualo o roble (Nothofagus obliqua (Mirb.) Oerst). Los bosques de hualo han sido sometidos a una fuerte extracción y reemplazo por plantaciones (Donoso 1993), manteniéndose la especie actualmente en poblaciones sujetas a un intenso proceso de fragmentación (Le-Quesne y Sandoval 2001). Este hecho motivó su inclusión como especie en estado de conservación vulnerable en la lista roja de especies amenazadas (González 1998).

En este contexto reviste especial importancia aportar con información que permita avanzar en el conocimiento de la estructura y el funcionamiento de los bosques de hualo en sus distintas áreas de distribución, con miras a su conservación y manejo sustentable (Fajardo y Alaback 2005). El presente estudio pretende aportar a la cuantificación de biomasa y a la estimación del carbono retenido en el mantillo y las raíces de un renoval de hualo en la precordillera andina de la VII Región del Maule.

\section{MÉTODOS}

Área de estudio. El estudio se desarrolló en un renoval de bosque nativo del tipo forestal Roble-Hualo en el Centro Experimental "El Picazo" ubicado en la precordillera andina de la comuna de San Clemente, Provincia de Talca de la

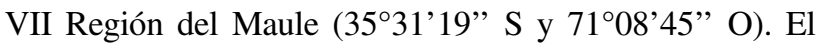
clima es templado cálido del tipo mediterráneo, con prolongada estación seca y lluvias invernales. En los meses fríos las temperaturas mínimas fluctúan entre $2^{\circ}$ y $3^{\circ} \mathrm{C}$, mientras que se alcanzan $23^{\circ}$ a $27^{\circ} \mathrm{C}$ en la época estival. Las precipitaciones varían entre 1.000 y $1.200 \mathrm{~mm}$ anuales, produciéndose en los meses de mayo a septiembre. El terreno se caracteriza por una topografía montañosa con pendientes poco pronunciadas (0 a 30\%) y altitudes que fluctúan entre 400 y $900 \mathrm{~m}$. Predominan los suelos trumaos (Andisoles) de textura franca a franca arcillosa en superficie, con mediana pedregosidad y erosión moderada (Arriagada 2004).

El renoval presenta en promedio 911 árboles ha-1 área basal de $10,8 \mathrm{~m}^{2} \mathrm{ha}^{-1}$ y diámetro medio cuadrático (DMC) de $12,3 \mathrm{~cm}$. El DAP fluctúa entre 7,3 y $22,5 \mathrm{~cm}$. El $99 \%$ de los árboles y del área basal corresponde a Nothofagus glauca y el restante a Lomatia hirsuta (Lam.) Diels ex Macbr. (cuadro 1).
Cuadro 1. Tabla de rodal del renoval de Roble-Hualo estudiado. Stand table of the studied young Roble-Hualo forest.

\begin{tabular}{|c|c|c|c|c|c|c|}
\hline \multirow{2}{*}{$\begin{array}{l}\text { DAP } \\
(\mathrm{cm})\end{array}$} & \multicolumn{2}{|c|}{ Nothofagus glauca } & \multicolumn{2}{|c|}{ Lomatia hirsuta } & \multicolumn{2}{|r|}{ Total } \\
\hline & $\mathrm{N}^{*}$ & $\begin{array}{l}\text { Área basal } \\
\left(\mathrm{m}^{2} \mathrm{ha}^{-1}\right)\end{array}$ & $\mathrm{N}$ & $\begin{array}{l}\text { Área basal } \\
\left(\mathrm{m}^{2} \mathrm{ha}^{-1}\right)\end{array}$ & $\mathrm{N}$ & $\begin{array}{c}\text { Área basal } \\
\left(\mathrm{m}^{2} \mathrm{ha}^{-1}\right)\end{array}$ \\
\hline 5 & 18,5 & 0,04 & - & - & 18,5 & 0,04 \\
\hline 10 & 538,9 & 4,23 & 5,6 & 0,04 & 544,5 & 4,27 \\
\hline 15 & 322,2 & 5,69 & - & - & 322,2 & 5,69 \\
\hline 20 & 25,9 & 0,81 & - & - & 25,9 & 0,81 \\
\hline Total & 905,5 & 10,77 & 5,6 & 0,04 & 911,1 & 10,81 \\
\hline
\end{tabular}

* Número de árboles ha $^{-1}$.

Colecta y procesamiento del mantillo y raíces. A fines de septiembre de 2005 se delimitaron en el renoval 10 parcelas de $1.000 \mathrm{~m}^{2}(20 \times 50 \mathrm{~m}$, corregidas en función de la pendiente). Las parcelas se dispusieron en dos hileras de cinco parcelas contiguas cada una, sobre una ladera alta con $30 \%$ a $45 \%$ de pendiente y exposición Sur. La ladera de forma y contorno convexos presenta una superficie irregular surcada por pequeñas hondonadas. En cada parcela se obtuvieron cinco muestras de mantillo, distanciadas a $10 \mathrm{~m}$ en el sentido de la pendiente, con un marco de $0,25 \mathrm{~m}^{2}(0,5 \times 0,5 \mathrm{~m})$, según lo recomendado por Sanquetta y Balbinot (2004). Se recolectaron en total 50 muestras que fueron trasladadas en bolsas de polietileno al laboratorio, donde se separó el mantillo en hojas y ramas.

En la posición de cada marco fueron extraídas muestras de suelo hasta los 5,10 y $20 \mathrm{~cm}$ de profundidad del perfil, utilizando un tubo metálico de $4,5 \mathrm{~cm}$ de diámetro interior (Bilbao 2005). Por debajo de los $20 \mathrm{~cm}$ comenzaba generalmente el estrato rocoso, por lo que se muestrearon sólo los $20 \mathrm{~cm}$ superiores del perfil. En los casos que la presencia de una raíz principal de un árbol impedía la extracción de la muestra, se tomaba un punto distante a $0,5 \mathrm{~m}$ sobre la línea de muestreo. Para cada profundidad se obtuvo un total de 50 muestras, las que fueron lavadas sobre un tamiz de $1 \mathrm{~mm}^{2}$ de apertura para extraer todas las raíces y clasificarlas según su diámetro en finas $(\leq 2$ $\mathrm{mm})$ y medianas $(2-5 \mathrm{~mm})$. La biomasa del mantillo y de las raíces se obtuvo al secar estos componentes en una estufa a $72^{\circ} \mathrm{C}$ por tres días y luego pesarlos en balanza analítica $(0,01 \mathrm{~g})$.

Diseño y análisis estadísticos. Se utilizó un diseño experimental en bloques aleatorios (10 bloques en el sentido de la pendiente) para el caso del mantillo, sometiéndose los valores a un análisis de varianza para detectar diferencias entre bloques. Para el caso de las raíces se utilizó un diseño factorial, considerando los factores profundidad de muestreo y diámetro de raíz. En este caso los valores no cumplían con los supuestos de normalidad y homocedasticidad de la varianza, por lo que se ensayaron las 
transformaciones propuestas por Kirk (1995), sin lograr cumplirse satisfactoriamente los supuestos del análisis de varianza. A raíz de ello se indagaron otras funciones matemáticas, lográndose un mejor ajuste utilizando el inverso de la función exponencial: $\mathrm{e}^{-\mathrm{x}}$ (siendo $\mathrm{x}=$ peso seco $^{0,975}$ para las profundidades $0-5$ y $5-10$ y $\mathrm{x}=$ peso $\operatorname{seco}^{1,5}$ para las profundidades $0-10$ y $\left.10-20\right)$. Los valores transformados se sometieron a un análisis factorial para detectar la influencia de cada factor y su interacción sobre la variación total de los datos.

La biomasa del mantillo y de las raíces fue multiplicada por 0,04 y por 6,29 respectivamente, para ser expresada en $\mathrm{Mg}$ por hectárea $\left(\mathrm{Mg} \mathrm{ha}^{-1}\right)$. El carbono acumulado se obtuvo al multiplicar la biomasa del mantillo y de las raíces por 0,44 , que corresponde al promedio ponderado de contenido de carbono determinado por Gayoso et al. (2002) para las especies nativas.

\section{RESULTADOS}

Debajo del renoval estudiado se cuantificó una acumulación de $12,47 \pm 4,08 \mathrm{Mg} \mathrm{ha}^{-1}$ de mantillo (cuadro 2), del cual una tercera parte correspondió a ramas y los restantes dos tercios a hojas. Las ramas se distribuyeron homogéneamente debajo del dosel, mientras que la distribución de las hojas evidenció una variación en sentido longitudinal $(P \leq 0,05)$ y transversal $(P \leq 0,01)$ a la pendiente. Sin embargo, no fue posible detectar una tendencia en función de la posición sobre la ladera (ladera baja vs. ladera alta), lo que da cuenta de una gran variabilidad en la distribución de las hojas.

Cuadro 2. Acumulación de biomasa $\left(\mathrm{Mg} \mathrm{ha}^{-1}\right)$ y almacenamiento de carbono $\left(\mathrm{Mg} \mathrm{ha}^{-1}\right)$ en mantillo y raíces. $\left.\mathrm{ha}^{-1}\right)$ in litter and roots.

Biomass accumulation $\left(\mathrm{Mg} \mathrm{ha}^{-1}\right)$ and carbon storage $(\mathrm{Mg}$

\begin{tabular}{llcc}
\hline \multicolumn{2}{c}{ Componentes } & $\begin{array}{c}\text { Biomasa }\left(\mathrm{Mg} \mathrm{ha}^{-1}\right) \\
\text { Media } \pm \mathrm{S}\end{array}$ & $\begin{array}{c}\text { Carbono* } \\
\left(\mathrm{Mg} \mathrm{ha}^{-1}\right)\end{array}$ \\
\hline Mantillo & ramas & $4,08 \pm 2,27$ & 1,80 \\
& hojas & $8,39 \pm 2,98$ & 3,69 \\
& Subtotal & $12,47 \pm 4,08$ & 5,49 \\
\multirow{2}{*}{ Raíces } & finas & $10,45 \pm 5,13$ & 4,60 \\
& gruesas & $10,54 \pm 5,75$ & 4,64 \\
\multirow{2}{*}{ Total } & Subtotal & $20,99 \pm 10,24$ & 9,24 \\
\hline
\end{tabular}

*Estimado con base en el promedio ponderado de contenido de carbono para especies nativas (Gayoso et al. 2002).

Hasta los $20 \mathrm{~cm}$ de profundidad del perfil se cuantificó una acumulación de raíces de 20,99 \pm 10,24 $\mathrm{Mg} \mathrm{ha}^{-1}$, aportando cada tipo de raíz la mitad a la biomasa total (cuadro 2). Más de la mitad de la biomasa de las raíces finas $(51 \%)$ se ubicó en el estrato superior del perfil (0-5 $\mathrm{cm}$ ), disminuyendo marcadamente con el aumento de profundidad (25 y 24\% para los estratos de 5-10 y 10-20 $\mathrm{cm}$, respectivamente) (figura 1). Por el contrario, sólo el $26 \%$ de la biomasa de raíces medianas fue encontrado en el estrato superior, manifestándose un leve incremento de su biomasa en el segundo estrato con un $29 \%$, mientras que el restante $45 \%$ se acumuló en el estrato más profundo. Teniendo en cuenta que el volumen de este último estrato correspondió al doble de volumen de suelo de cada uno de los dos estratos superiores, se desprende que las raíces medianas se repartieron más uniformemente en profundidad que las raíces finas.

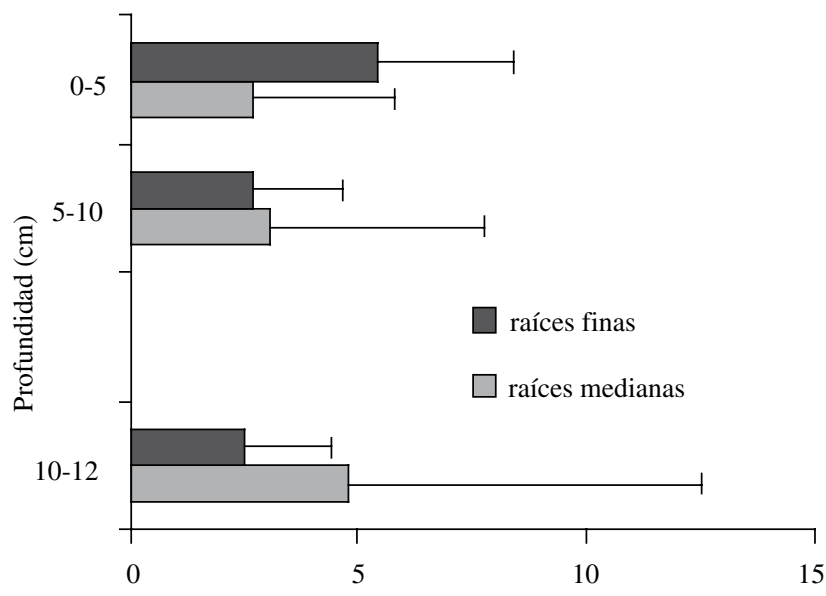

Figura 1. Distribución de la biomasa de raíces finas $(\leq 2 \mathrm{~mm})$ y raíces medianas $(2-5 \mathrm{~mm})$ en distintos estratos de profundidad del perfil. Líneas muestran la desviación estándar.

Biomass distribution of fine roots $(\leq 2 \mathrm{~mm})$ and medium roots $(2-5 \mathrm{~mm})$ in different soil layers.

Considerando la biomasa acumulada hasta los primeros $10 \mathrm{~cm}$ y entre los 10-20 cm, se evidenció a mayor profundidad una disminución de ambos tipos de raíces, pero más marcada $(P \leq 0,01)$ para las raíces finas. La interacción significativa entre los factores profundidad y diámetro de raíz $(P \leq 0,01)$ da cuenta de la distribución diferencial de las raíces de distinto grosor. De igual manera, se evidenció una interacción significativa entre ambos factores $(P \leq 0,01)$ para el caso de los estratos más superficiales $(0-5$ y $5-10 \mathrm{~cm})$. Esto se explica por una mayor acumulación de biomasa de raíces finas que de raíces medianas $(P \leq 0,01)$ en el estrato más superficial $(0-5 \mathrm{~cm})$, a la par de manifestar las primeras una marcada disminución $(P \leq 0,01)$ en el estrato de $5-10 \mathrm{~cm}$. Por el contrario, las raíces medianas se distribuyeron más homogéneamente entre ambos estratos superiores.

La biomasa total acumulada originó una absorción de $14,78 \mathrm{Mg} \mathrm{ha}^{-1}$ de carbono (cuadro 2), equivalente a 54,4 $\mathrm{Mg} \mathrm{ha}{ }^{-1}$ de $\mathrm{CO}_{2}$ no emitidas a la atmósfera. 


\section{DISCUSIÓN}

La gran variabilidad en la distribución del componente hojas del mantillo pudo deberse a varios factores. Por un lado, posiblemente existió un aporte no uniforme de la hojarasca, siendo mayor debajo de las copas de los árboles (Schlatter et al. 2006). Por otro lado, los troncos de los árboles y la vegetación del sotobosque pueden haber actuado como barreras, frenando el arrastre superficial causado por la escorrentía y el viento (Fahnestock et al. 2000). A este efecto puede haberse sumado la irregularidad del terreno, acumulándose el mantillo en las pequeñas hondonadas que surcan la superficie del terreno. Posiblemente, debido a su mayor tamaño y peso individual, comparado al de las hojas, el componente ramas del mantillo estuvo menos expuesto a un reordenamiento posterior a su desprendimiento.

El mantillo correspondió al tipo de humus mull (Schlatter et al. 2003), por la presencia de un horizonte $\mathrm{O}_{\mathrm{i}}$ suelto de $3 \mathrm{a} 5 \mathrm{~cm}$ de espesor y por la ausencia de un horizonte $\mathrm{O}_{\mathrm{e}}$. Es un mantillo originado principalmente por hojarasca de una especie latifoliada caducifolia (el hualo), por lo que su acumulación dependerá de la época de muestreo, siendo el valor obtenido en este estudio sólo válido a nivel referencial de fines de invierno.

Hasta los $20 \mathrm{~cm}$ de profundidad del perfil se acumuló similar biomasa para los dos rangos de grosor de raíces considerados, pero diferencialmente distribuida en profundidad. Esta distribución de las raíces de distinto grosor tiene relación con la función específica de las mismas, siendo las raíces más finas las más eficientes en absorción de agua y nutrientes, cuya disponibilidad es mayor en los estratos más superficiales (Claus y George 2005). Los ecosistemas forestales mayoritariamente presentan este tipo de distribución de raíces finas, es decir una disminución drástica de su biomasa en profundidad. Jackson et al. (1997) reportan un promedio de acumulación del 83 y $79 \%$ del total de las raíces finas vivas en los $30 \mathrm{~cm}$ superiores del perfil de suelo en bosques boreales y esclerófilos, respectivamente. Para los bosques templados deciduos los mismos autores informan una acumulación del $63 \%$ en los $30 \mathrm{~cm}$ superiores del perfil, mientras que los bosques de coníferas y los bosques tropicales se caracterizan por un sistema radical fino que profundiza más ( $45 \%$ del total de las raíces finas vivas es reportado para los $30 \mathrm{~cm}$ superiores en el caso de las coníferas y 42 a $57 \%$ en los bosques tropicales deciduos y perennes respectivamente). Yanai et al. (2006) en cinco rodales de bosque mixto caducifolio de diferentes edades encontraron un patrón de distribución radical similar al encontrado para este estudio, una marcada disminución de las raíces finas en profundidad y una disminución más atenuada para las raíces de mayor diámetro.

Jackson et al. (1997) encontraron una biomasa de raíces finas promedio de $0,78 \mathrm{~kg} \mathrm{~m}^{-2}$ en bosques templados deciduos, lo que es un valor inferior al de este estudio, 1,05 $\mathrm{kg} \mathrm{m}^{-2}$ para sólo los $20 \mathrm{~cm}$ superiores del perfil; también citan una biomasa promedio de $0,44 \mathrm{~kg} \mathrm{~m}^{-2}$ para raíces finas vivas, coincidiendo con mediciones posteriores de otros autores. Leuschner et al. (2004), comparando la biomasa y morfología de raíces finas en un gradiente de fertilidad y humedad para bosques antiguos de Fagus sylvatica L., encontraron una acumulación de 0,32 a $0,47 \mathrm{~kg} \mathrm{~m}^{-2}$ de raíces vivas en los $40 \mathrm{~cm}$ superiores de los distintos sitios y de sólo $0,14 \mathrm{~kg} \mathrm{~m}^{-2}$ para el sitio más seco. La necromasa de raíces finas osciló entre 3,50 y $0,40 \mathrm{~kg} \mathrm{~m}^{-2}$. A su vez, Claus y George (2005) encontraron una acumulación de raíces finas vivas hasta los $30 \mathrm{~cm}$ de profundidad de 0,53 y $0,64 \mathrm{~kg} \mathrm{~m}^{-2}$ en rodales jóvenes de Fagus sylvatica (15 y 30 años, respectivamente) y de 0,24 y $0,33 \mathrm{~kg}$ $\mathrm{m}^{-2}$ en rodales adultos (62 y 111 años, respectivamente). Moreno-Chacón y Lusk (2004) reportaron una acumulación de 4,69 $\mathrm{kg} \mathrm{m}^{-2}$ de raíces finas vivas hasta $\operatorname{los} 80 \mathrm{~cm}$ de profundidad en rodales antiguos de selva valdiviana donde Nothofagus dombeyi (Mirb.) Oerst. era la especie emergente, aduciendo que se muestrearon dirigidamente sitios de alta densidad.

Teniendo en cuenta que en el presente estudio no se diferenciaron las raíces vivas de las muertas y que se tomaron muestras sólo hasta los $20 \mathrm{~cm}$ de profundidad del perfil, los valores alcanzados calzan dentro del rango informado en la literatura. Según Cronan (2003), estimaciones de biomasa radical basada en muestreos arrojarían diferentes resultados dependiendo de la metodología utilizada, tanto referido al tamaño de la muestra, a la profundidad de muestreo, como a la época del año en que se extrae la muestra, sumándose a estas variaciones el error de procesamiento de la muestra. Esta afirmación arroja luz sobre la necesidad de realizar un muestreo más exhaustivo del sistema radical, teniendo en cuenta diferentes épocas de muestreo y la totalidad del perfil explorado, ya que el estrato rocoso comenzaba a partir de $\operatorname{los} 20 \mathrm{~cm}$ y a profundidades variables, lo que en el presente estudio podría haberse traducido en una subestimación no ponderada de la biomasa. A pesar de ser las raíces de menor tamaño y rápido recambio las responsables del mayor aporte e incorporación de carbono a los suelos forestales (Matamala et al. 2003), sería además recomendable cuantificar el sistema radical principal, que representa el mayor compartimiento de la biomasa subterránea de los ecosistemas forestales (Gayoso et al. 2002, Geldres et al. 2006). Por último, y considerando que a nivel global los suelos acumulan la mayor parte del carbono fijado en los ecosistemas (Brady y Weil 1996, Schumacher et al. 2004, Schumacher y Witschoreck 2004), debería medirse el contenido de materia orgánica del suelo para poder estimar el real aporte del subsistema subterráneo de los renovales de Roble-Hualo a la retención de carbono.

\section{AGRADECIMIENTOS}

Los autores agradecen las valiosas sugerencias de los evaluadores anónimos y del editor. 


\section{REFERENCIAS}

Arriagada P. 2004. Evaluación de impacto ambiental del plan de actividades de la Estación Experimental "El Picazo". Memoria de Título. Talca, Chile. Universidad de Talca. 55 p.

Bilbao N. 2005. Estimación de biomasa radical acumulada bajo un bosque nativo del tipo forestal Roble-Hualo de la estación experimental "El Picazo", de la comuna de San Clemente, VII Región. Memoria de Título. Talca, Chile. Universidad de Talca. 58 p.

Brady N, R Weil. 1996. Soil Organic Matter. In The Nature and Properties of Soils. Prentice-Hall, U.S.A. p. 361-399.

Claus A, E George. 2005. Effect of stand age on fine-root biomass and biomass distribution in three European forest chronosequences. Can. J. For. Res. 35: 1617-1625.

CONAF - CONAMA (Corporación Nacional Forestal, CL - Comisión Nacional del Medio Ambiente, CL). 1999. Catastro y evaluación de recursos vegetacionales nativos de Chile. Informe Regional Séptima Región. Santiago, Chile. Corporación Nacional Forestal - Comisión Nacional del Medio Ambiente. 116 p.

Cronan C. 2003. Belowground biomass, production, and carbon cycling in mature Norway spruce, Maine, U.S.A. Can. J. For. Res. 33: 339-350.

Donoso C. 1981. Tipos forestales de los bosques nativos de Chile. Santiago, Chile. CONAF, FAO, FO:DP/CHI/76/003. Documento de Trabajo $\mathrm{N}^{\circ} 38$. Corporación Nacional Forestal. 83 p.

Donoso C. 1993. Bosques templados de Chile y Argentina. Variación, Estructura y Dinámica. Santiago, Chile. Editorial Universitaria. $483 \mathrm{p}$.

Ellsworth D. 1999. $\mathrm{CO}_{2}$ enrichment in a maturing pine forest: are $\mathrm{CO}_{2}$ exchange and water status in the canopy affected? Plant, Cell and Environment 22: 461-472.

Fahnestock J, K Povirk, J Welter. 2000. Ecological significance of litter redistribution by wind and snow in artic landscapes. Ecography 23: 623-631.

Fajardo A, P Alaback. 2005. Effects of natural and human disturbances on the dynamics and spatial structure of Nothofagus glauca in south-central Chile. J. Biogeogr. 32: 1811-1825.

Gayoso J, J Guerra, D Alarcón. 2002. Contenido de carbono y funciones de biomasa en especies nativas y exóticas. Universidad Austral de Chile. Valdivia, Chile. Consultado 20 septiembre de 2006. Disponible en: http://www.uach. $\mathrm{cl} /$ procarbono/Documentos/Informe $\% 20$ Tecnico $\% 20$ Biomasa.pdf

Gayoso J, J Guerra. 2005. Contenido de carbono en la biomasa aérea de bosques nativos de Chile. Bosque 26(2): 33-38.

Geldres E, V Gerding, J Schlatter. 2006. Biomasa de Eucalyptus nitens de 4-7 años de edad en un rodal de la X Región, Chile. Bosque 27(3): 223-230.

González M. 1998. Nothofagus glauca. In IUCN 2007. 2007 IUCN Red List of Threatened Species. Consultado 16 octubre de 2007. Disponible en: http://www.iucnredlist.org

Groninger J, J Seiler, S Zedaker, P Berrang. 1995. Effects of elevated $\mathrm{CO}_{2}$, water stress and nitrogen level on competitive interactions of simulated loblolly pine and sweetgum stands. Can. J. For. Res. 25: 1077-1083.

Guerra J. 2001. Evaluación de la biomasa radical gruesa en la especie Nothofagus alpina (Poepp. et Mol) Oerst (Raulí) en la provincia de Malleco. Tesis Ing. Forestal. Valdivia, Chile. Universidad Austral de Chile. 58 p.

Hamilton J, R Thomas, E De Lucia. 2001. Direct and indirect effects of elevated $\mathrm{CO}_{2}$ on leaf respiration in a forest ecosystem. Plant, Cell and Environment 24: 975-982.

IPCC (Intergovernmental Panel on Climate Change). 2001. Climate Change 2001: Synthesis Report, Summary for Policymakers. This summary, approved in detail at IPCC Plenary XVIII Wembley, United Kingdom, 24-29 September. 34 p. Consultado 10 de septiembre 2007. Disponible en: http://www.ipcc.ch/pub/SYRspm.pdf

Jackson R, H Mooney, E Schulze. 1997. A global budget for fine root biomass, surface area, and nutrient contents. Proc. Natl. Acad. Sci. USA (94): 7362-7366.

Kirk R. 1995. Experimental design: procedures for the behavioral sciences. 3rd ed. Brooks/Cole Publishing Company and International Thompson Publishing Company. USA. $921 \mathrm{p}$.

Koning F De, R Olschewski, E Veldkamp, P Benítez, P Laclau, M López, M de Urquiza, T Schlichter. 2002. Evaluation of the $\mathrm{CO}_{2}$ sequestration potential of afforestation projects and secondary forests in two different climate zones of South America. Eschborn, Alemania. Deutsche Gesellschaft für Technische Zusammenarbeit (GTZ) GmbH. 149 p.

Le-Quesne C, L Sandoval. 2001. Extensión del límite sur para Nothofagus glauca (Phil.) Krasser. Gayana Bot. 58(2): 139-142.

Leuschner C, D Hertel, I Schmidt, O Koch, A Muhs, D Hölscher. 2004. Stand fine root biomass and fine root morphology in old-growth beech forests as a function of precipitation and soil fertility. Plant and Soil 258: 43-56.

Luan, J, Y Luo, J Reynolds. 1999. Responses of loblolly pine ecosystem to $\mathrm{CO}_{2}$ enrichment: a modeling analysis. Tree Physiology 19: 279-287.

Matamala R, M González-Meler, J Jastrow, R Norby, W Schlesinger. 2003. Impacts of Fine Root Turnover on Forest NPP and Soil C Sequestration Potencial. Science 302: 1385-1387.

Moreno-Chacón M, C Lusk. 2004. Vertical distribution of fine root biomass of emergent Nothofagus dombeyi and its canopy associates in a Chilean temperate rainforest. Forest Ecology and Management 199: 177-181.

Myers D, R Thomas, E De Lucia. 1999. Photosynthetic capacity of loblolly pine (Pinus taeda L.) trees during the first year of carbon dioxide enrichment in a forest ecosystem. Plant, Cell and Environment 22: 473-481.

Rasse D, B Longdoz, R Ceulemans. 2001. TRAP: a modeling approach to below-ground carbon allocation in temperate forests. Plant and Soil. 229: 281-293.

Sánchez F, R Eaton. 2001. Sequestering Carbon and Improving Soils: Benefits of Mulching and Incorporating Forest Slash. Journal of Forestry 99: 32-36.

Sanquetta R, R Balbinot. 2004. Métodologias para determinação de biomassa florestal. In Sanquetta R, R Balbinot, M Ziliotto eds. Fixação de carbono: atualidades, projetos e pesquisas. Curitiba, Brasil. AM Impresos. p. 77-93.

Schlatter J, R Grez, V Gerding. 2003. Manual para el Reconocimiento de Suelos. Valdivia, Chile. Imp. Universidad Austral de Chile. 114 p.

Schlatter J, V Gerding, S Calderón. 2006. Aporte de la hojarasca al ciclo biogeoquímico en plantaciones de Eucalyptus nitens, X Región, Chile. Bosque 27(2): 115-125. 
Schumacher M, F König, J Kleinpaul. 2004. Quantificação de carbono orgânico na serapilheira, sub-bosque e solo de uma floresta de Pinus elliotii Engelm. aos 36 anos em Santa Maria, RS. In Sanquetta R, R Balbinot, M Ziliotto eds. Fixação de carbono: atualidades, projetos e pesquisas. Curitiba, Brasil. AM Impresos. p.125-132.

Schumacher M, R Witschoreck. 2004. Inventario de carbono em povoamentos de Eucalyptus ssp. Nas propriedades fumageiras do sul do Brasil: "um estudo de caso". In Sanquetta R, R Balbinot, M Ziliotto eds. Fixação de carbono: atualidades, projetos e pesquisas. Curitiba, Brasil. AM Impresos. p. 111-124.

Telewski F, R Swanson, B Strain, J Burns. 1999. Wood properties and ring width responses to long-term atmospheric $\mathrm{CO}_{2}$ enrichment in field-grown loblolly pine (Pinus taeda L.). Plant, Cell and Environment 22: 213-219.
Teskey R. 1997. Combined effects of elevated $\mathrm{CO}_{2}$ and air temperature on carbon assimilation of Pinus taeda trees. Plant, Cell and Environment 20: 373-380.

Tissue D, R Thomas, B Strain. 1997. Atmospheric $\mathrm{CO}_{2}$ enrichment increases growth and photosynthesis of Pinus taeda: a 4 year experiment in the field. Plant, Cell and Environment 20: 1123-1143.

Vogt K, D Vogt, J Bloomfield. 1998. Analysis of some direct and indirect methods for estimating root biomass and production of forests at an ecosystem level. Plant and Soil 200: 71-89.

Yanai R, B Park, S Hamburg. 2006. The vertical and horizontal distribution of roots in northern hardwood stands of varying age. Can. J. For. Res. 36: 450-459.

Recibido: 19.12 .06

Aceptado: 10.12.07 